\title{
Calidad microbiológica de la materia prima y el producto final del ceviche de tilapia y de camarón expendidos en el Ârea Metropolitana de San José, Costa Rica
}

\author{
María Laura Arias Echandi y Carolina Chaves Ulate \\ Centro de Investigación en Enfermedades Tropicales, Universidad de Costa Rica,Facultad de Microbiologia, Universidad de Costa Rica; \\ maria.ariasechandi@ucr.ac.cr
}

Recibido 29-XI-2011 Corregido 18-I-2012 Aceptado 28-II-2012

\begin{abstract}
Microbiological quality of raw material and final product of tilapia and shrimp ceviche expended in the Metropolitan Area of San José, Costa Rica. Ceviche is a common use dish from Latin American countries, made up of raw fish or seafood marinated with lemon or lime juice, and several seasoning ingredients. It includes the normal flora coming from raw product and flora acquired from handling and additional ingredients. The product is consumed raw, representing a risk for Public Health. The aim of this work was to determine the microbiological quality of tilapia fish ceviche and shrimp ceviche sold at the Metropolitan Area of San José, Costa Rica, as well as the raw material used in its elaboration. A total of 25 samples of tilapia ceviche and 27 samples of shrimp ceviche were analyzed; the same number of raw material was analyzed. The behavior of some of the spoilage populations was analyzed in 25 samples of shrimp and same number of tilapia ceviche during its storage at $4{ }^{\circ} \mathrm{C}$. Even though the ceviche includes manipulation plus an important bacterial contamination in raw material, there is an important reduction in the bacterial charge of the final product, especially referring to total aerobic psychrofilic plate count and total coliforms; but not to fecal coliforms. Escherichia coli was isolated from 15\% of the tilapia's ceviche and $4 \%$ from shrimp's ceviche, 3 strains of Listeria monocytogenes were isolated from tilapia, one from shrimp, 3 strains of $V$. parahaermolyticus were isolated from shrimp and 2 from its ceviche. Salmonella spp. was not isolated. The $\mathrm{pH}$ of ceviche plates stored at $4^{\circ} \mathrm{C}$ for one week showed a trend towards stability or a slight increase of this parameter. The concentration of spoilage microorganisms suffered slight variations, nevertheless, the low number of Pseudomonas spp. present in tilapia's ceviche is an outstanding result considering that this population is associated to spoilage of the product.
\end{abstract}

\section{KEY WORDS}

Ceviche, tilapia, shrimp, spoilage.

\section{RESUMEN}

El ceviche es un platillo formulado a base de pescado de agua salada o dulce, o mariscos frescos y diversos aliños. Su elaboración acarrea no solo la flora normal y adquirida de la materia prima a utilizar, sino que también la flora que se agregue durante su procesamiento y a partir de ingredientes adicionales. Además, es un producto que se consume marinado, representando un riego para la salud pública. El objetivo de este trabajo fue determinar la calidad microbiológica del ceviche de tilapia y del ceviche de camarón que se vende en el Área Metropolitana de San José, Costa Rica, así como de la materia prima utilizada en su respectiva elaboración. Para tal efecto, se analizaron 25 muestras de ceviche de tilapia y 27 de camarón, analizando igual número de muestras de materia prima. De la misma manera, se analizó el comportamiento de las poblaciones de algunos microorganismos de deterioro a partir de 25 muestras de ceviche de camarón y 25 muestras de ceviche de tilapia durante su almacenaje a $4^{\circ} \mathrm{C}$. A pesar de la contaminación bacteriológica presente en la materia prima, y de que el ceviche es un producto que incluye mucha manipulación, existe una disminución importante en las cargas bacterianas en el producto final, especialmente en lo que respecta a recuento total de aerobios psicrófilo y coliformes totales, no así los coliformes fecales. Se aisló Escherichia coli a partir de 15\% de ceviches de tilapia y $4 \%$ de los ceviches de camarón, 3 cepas de Listeria monocytogenes a partir de tilapia, 1 a partir de camarón, y 3 cepas de $V$. parahaermolyticus a partir de camarón y 2 a partir de su ceviche. No se aisló Salmonella spp. En cuanto a los ceviches almacenados a $4^{\circ} \mathrm{C}$ por una semana, cabe destacar que el $\mathrm{pH}$ mostró una tendencia hacia la estabilidad o de aumento muy ligero. De la misma manera, la concentración de microorganismos de deterioro sufrió pocas variaciones, no obstante, llama la atención el bajo número de Pseudomonas spp. presentes en los ceviches de tilapia.

PALABRAS CLAVE

Ceviche, tilapia, camarón, deterioro. 
El ceviche es un platillo formulado a base de pescado de agua salada o dulce, o mariscos frescos y diversos aliños que forman parte de la gastronomía de varios países Latinoamericanos.

La tilapia (Oreochromis niloticus), es uno de los peces que actualmente tiene mayor aceptación por parte de los consumidores y es considerada una atractiva opción del menú en cadenas de restaurantes a nivel nacional e internacional, aparte de su utilización en la elaboración de ceviche (Chacón 2002). En términos de volumen de producción, Taiwán es el mayor exportador del mundo y junto con Tailandia e Indonesia constituyen los mayores proveedores de filetes de tilapia congelados a los Estados Unidos mientras que Costa Rica domina el mercado de tilapia fresca a ese país (Víquez et al. 2003).

Desde principio de la década de los setenta, se ha promovido en Costa Rica el desarrollo de la acuicultura siendo casi totalmente dominada por el tipo continental de agua dulce, con énfasis en el cultivo de peces, específicamente trucha y tilapia (Ramírez 2002). El cultivo de esta última se da en las siete provincias del país, no obstante, Guanacaste es la provincia con mayor producción (30 productores con 7215 toneladas/año) y Alajuela, específicamente San Carlos, posee el mayor número de productores (435 con 444 toneladas/año) (Otálora 2002).

Con respecto a la flora microbiana de la tilapia viva, ésta depende de la flora existente en las aguas de donde proviene (Pascual et al. 2000) y varía de acuerdo con el hábitat de la especie, sobre todo con la temperatura, profundidad y grado de contaminación de las aguas. Se destaca la presencia de psicrófilos Gram negativos incluyendo Pseudomonas, Shewanella, Moraxella, Acinetobacter, Flavobacterium y Aeromonadaceae. Entre los Gram positivos existe una proporción variable de Bacillus, Micrococcus, Clostridium, Lactobacillus y Corynebacterium (Pascual et al. 2000, Morales et al. 2004). Las bacterias patógenas o indicadoras de contaminación raramente son encontradas en el pescado recién capturado, a no ser que provenga de aguas excesivamente contaminadas con materia fecal (Pascual et al. 2000).

Por otro lado, el camarón también forma parte de la materia prima utilizada en la elaboración de ceviches. El camarón es un crustáceo del orden de los decápodos. Viven tanto en aguas dulces como saladas, así como en regiones templadas y tropicales o frías y gélidas. Habita en aguas poco profundas, cerca del fondo, donde se alimenta de plantas y pequeños animales. Ciertas especies son pelágicas y viven en aguas abiertas, a veces a profundidades de hasta 5 kilómetros (Pascual et al. 2000).

En Costa Rica, el cultivo de crustáceos es todavía una actividad poco desarrollada y la mayoría de proyectos de camarón se desarrollan en aguas salobres. De acuerdo con datos del Instituto Costarricense de Pesca y Acuicultura (INCOPESCA), existen unas 1500 hectáreas dedicadas a la producción de camarón en todo el país, con una concentración de la actividad en la costa pacífica. Además, hay alrededor de 118 camaroneros, que representan un $7 \%$ del total de acuicultores del país, la a mayor parte de ellos son pequeños productores del Golfo de Nicoya (Otárola 2002).

La microflora de los crustáceos es semejante a la de los pescados recién capturados, está formada principalmente por Pseudomonas, Achromobacter, Micrococcus y Corynebacterium; pero también se pueden encontrar Flavobacterium, Alteromonas, Moraxella, Escherichia, Proteus, Serratia, Bacillus, Vibrio, Clostridium, hongos filamentosos y levaduras. Debido a que es un alimento tan perecedero, se conserva desde su captura a bajas temperaturas, lo que determina que la proporción de Acinetobacter/Moraxella en algunos casos sea del $80 \%$. Entre las bacterias que participan en la descomposición de estos alimentos se encuentran Pseudomonas, Shewanella, Alteromonas y especies de Enterobacteriaceae (Pascual et al. 2000).

La elaboración del ceviche acarrea no solo la flora normal y adquirida de la materia prima a utilizar, sino que también la flora que se agregue durante su elaboración y a partir de ingredientes adicionales. Aparte de esto, es un producto que se consume únicamente marinado, representando un riesgo para la salud.

Diversos géneros patógenos pueden contaminar el ceviche, incluyendo entre estos, Salmonella spp. y Listeria monocytogenes, entre otros (Shen et al. 2006).

Con base en lo anterior, en este estudio se determinó la calidad microbiológica del ceviche de pescado (tilapia) y del ceviche de camarón de mar que se vende en el Área Metropolitana de San José, Costa Rica, así como de la materia prima utilizada en su respectiva elaboración. También, se analizó el comportamiento de las poblaciones de microorganismos de deterioro del ceviche, a través de su vida de almacenaje a temperatura de refrigeración. Se pretende con lo anterior, valorar la variación en la flora bacteriana presente en tilapia y camarón debido a su manipulación, procesamiento y almacenamiento, en aras de establecer si su producto final representa un riesgo para la Salud Pública.

\section{METODOLOGÍA}

\section{Localización del proyecto}

El estudio se llevó a cabo en el Laboratorio de Microbiología de Alimentos de la Facultad de Microbiología, Universidad de Costa Rica, durante los meses de enero a julio, 2011. 


\section{Origen de las muestras}

Para cada ceviche analizado $(n=25$ para tilapia y 27 para camarón), se evaluó igual número de muestras de materia prima. Las muestras provienen de pescaderías y supermercados ubicados en el Área Metropolitana de San José, Costa Rica.

\section{Análisis}

Para las 25 muestras de tilapia (materia prima del ceviche) los análisis realizados incluyeron el recuento total de microorganismos aerobios psicrófilos, recuento de coliformes totales, fecales y Escherichia coli, presencia/ausencia de Listeria monocytogenes y de Salmonella spp. en $25 \mathrm{~g}$.

Para las 27 muestras de camarón pelado (materia prima del ceviche) se realizaron los análisis listados anteriormente para tilapia así como la presencia/ausencia de Vibrio cholerae en $25 \mathrm{~g}$ y el Número Más Probable de $V$. parahaemolyticus.

Las muestras de ceviche de tilapia y de camarón fueron evaluadas realizando el recuento total de aerobios psicrófilos, el NMP de coliformes totales, fecales y de E. coli así como la presencia/ausencia de los patógenos citados anteriormente.

Para el análisis del comportamiento de algunas de las cepas de deterioro asociadas a ceviche, se evaluaron 25 muestras de ceviche de camarón empacado y 25 muestras de ceviche de tilapia empacado almacenadas a lo largo de una semana a $4^{\circ} \mathrm{C}$. A cada muestra se le realizó una medición de su pH y se cuantificó la población de Pseudomonas sp., Vibrio sp. y enterococos presentes los días 0,2 y 5.

Recuento total de aerobios psicrófilos: Se realizó según la metodología de Pouch (2001). Se pesaron $10 \mathrm{~g}$ de cada muestra y se diluyeron en $90 \mathrm{~mL}$ de agua peptonada estéril 0,1\% (APE). Se realizaron diluciones decimales seriadas hasta $10^{-7}$ en APE $0,1 \%$ y a partir de cada una se inocularon, por vaciado, platos de Agar Estándar + TTC (2,3,5 cloruro de trifeniltetrazolium) que se incubaron a $7^{\circ} \mathrm{C}$ por 6 días en atmósfera aerobia.

Recuento de coliformes totales, fecales y Escherichia coli: Se realizó según la metodología de Pouch (2001). A partir de las diluciones previamente preparadas se determinó el recuento en placa de coliformes utlizando Agar Bilis Rojo Violeta. Para la detección de coliformes totales, se incubaron las placas a $35^{\circ} \mathrm{C}$ por $48 \mathrm{~h}$, para la detección de coliformes fecales, se incubaron a $44,5^{\circ} \mathrm{C}$ por $24 \mathrm{~h}$. Para la determinación de Escherichia coli, se realizó, a partir de los coliformes fecales, una prueba de indol incubada a $44,5^{\circ} \mathrm{C}$ por $24 \mathrm{~h}$.
NMP/g de coliformes totales y fecales: Se empleó la técnica de Número Más Probable (NMP) descrita por Pouch (2001) utilizando Caldo Lactosado simple con campana Durham en la fase de preenriquecimiento $\left(35^{\circ} \mathrm{C}, 48 \mathrm{~h}\right)$, Caldo Bilis Verde Brillante (CBVB), incubado $48 \mathrm{~h}$ a $35^{\circ} \mathrm{C}$ para la confirmación de coliformes totales y caldo Escherichia coli (EC), incubado $24 \mathrm{~h}$ a $44,5^{\circ} \mathrm{C}$ para la confirmación de coliformes fecales. E. coli fue confirmada utilizando la prueba de indol.

Aislamiento de Salmonella spp. (FDA 1995): Se siguió el método descrito en el Manual del Bacteriología de la FDA, 1995. Se pre-enriquecieron $25 \mathrm{~g}$ de cada muestra en 225 $\mathrm{mL}$ de agua peptonada estéril (APE) $0,1 \%$, incubando $24 \mathrm{~h}$ a $35^{\circ} \mathrm{C}$. El enriquecimiento selectivo se realizó utilizando caldo tetrationato y caldo selenito, incubando $24 \mathrm{~h} \mathrm{a} 43^{\circ} \mathrm{C}$ y $35^{\circ} \mathrm{C}$ respectivamente. El aislamiento selectivo se realizó en los agares xilosa lisina desoxicolato (XLD) y Hecktoen, incubando $24 \mathrm{~h}$ a $35^{\circ} \mathrm{C}$. La confirmación bioquímica de las colonias sospechosas se realizó utilizando el API 20E y la confirmación serológica usando anticuerpos monoclonales.

Aislamiento de Listeria monocytogenes (FDA 1995): Se siguió la metodología descrita en el Manual de Bacteriología de la FDA, 1995 para determinar la presencia/ausencia de esta bacteria en $25 \mathrm{~g}$. Brevemente, $25 \mathrm{~g}$ de cada muestra fueron enriquecidos en $225 \mathrm{~mL}$ de caldo de Listeria e incubados a $30^{\circ} \mathrm{C}$ por $48 \mathrm{~h}$. Posteriormente, se inocularon placas de agar Oxford a partir del caldo del enriquecimiento, las cuales fueron incubadas por 48 horas a $35^{\circ} \mathrm{C}$. Las colonias típicas fueron confirmadas por la prueba de luz de Henry, morfología, motilidad, hemólisis, prueba de CAMP (Christie, Atkins and Much-Petersen) y utilización de xilosa y ramnosa.

Presencia/ ausencia de Vibrio cholerae: Siguiendo la metodología descrita por Pouch (2001), 25g de cada muestra fueron enriquecidos en $225 \mathrm{~mL}$ agua peptonada alcalina estéril por 8 y $24 \mathrm{~h}$ a $35^{\circ} \mathrm{C}$. Una asada de este enriquecimiento fue inoculada en agar tiosulfato citrato bilis sacarosa (TCBS) e incubada a $35^{\circ} \mathrm{C}$ por $24 \mathrm{~h}$. Las colonias sospechosas fueron identificadas mediante pruebas bioquímicas.

Aislamiento de V. parahaemolyticus: Siguiendo la metodología descrita por Pouch (2001), a partir de las diluciones previamente preparadas se inocularon series de 3 tubos de Agua peptonada estéril (APE) $0,1 \%+3 \% \mathrm{NaCL}$, los cuales fueron incubados a $35^{\circ} \mathrm{C}$ durante 24 horas. Los tubos que mostraron turbidez fueron inoculados por rayado en agar TCBS e incubados a $35^{\circ} \mathrm{C}$ durante 24 horas ; los cuales fueron incubados a $35^{\circ} \mathrm{C}$ durante 24 horas. Los 
tubos que mostraron turbidez fueron inoculados por rayado en agar TCBS e incubados a $35^{\circ} \mathrm{C}$ durante 24 horas.

Recuento de Enterococcus spp.: Se siguió la metodología descrita por Pouch (2001). A partir de cada muestra se prepararon diluciones decimales tal y como se describió anteriormente. Se rayó una asada de cada dilución en agar KF. Las placas se incubaron por 48 horas a $35^{\circ} \mathrm{C}$, con el fin de aumentar la selectividad. Las colonias que crecieron en el agar etil violeta (EVA) se evaluaron por: tinción de Gram (cocos gram positivos), catalasa (negativa), crecimiento en CTS con $6,5 \%$ de $\mathrm{NaCl}$ (positivo) y crecimiento en presencia de bilis con reducción de la esculina (positivo)

Recuento de Pseudomonas sp.: Siguiendo la metodología descrita en Pouch (2001), se rayó 0,1 mL de cada una de las diluciones previamente preparadas en agar cetrimida, el cual fue incubado a $35^{\circ} \mathrm{C}$ ambiente por 2 días.

Identificación bacteriana: Las cepas aisladas, fueron identificadas utilzando el Vitek II (Biomereiux).

Medición de pH: Se utilizó un pH-metro Cole Parmer 440 para la medición del $\mathrm{pH}$ de los ceviches evaluados.

\section{RESULTADOS}

En los cuadros 1 y 2 se compara el recuento total de aerobios psicrófilos y el recuento de coliformes totales y fecales presentes en la materia prima y en los respectivos productos finales.

En tilapia, 92\% de la materia prima presentó un recuento total de aerobios psicrófilos superior a $1000 \mathrm{UFC} / g$, únicamente el $43 \%$ de las muestras de ceviche alcanzaron este parámetro. De la misma manera, el 92\% de la materia prima presentó coliformes totales (>10UFC/g), contrastando con un $64 \%$ de positividad del ceviche.

Un segundo parámetro contemplado por la ICMSF para pescado crudo establece un límite máximo de coliformes fecales de 5,0 x 102 UFC/g (9). En el presente trabajo, se encuentra que $20 \%$ de las muestras de materia prima de tilapia presentaron límites superiores a los referidos. De la misma manera, 52\% de las muestras de ceviche de tilapia presentaron coliformes fecales.

Se aislaron seis cepas E. coli a partir de la tilapia y a partir de los ceviches respectivos únicamente cuatro cepas. También, se aislaron, a partir de la tilapia, tres cepas de Listeria monocytogenes, las cuales no fueron detectadas

CUADRO 1

Comparación del recuento total psicrófilo aerobio, coliformes totales y coliformes fecales presentes en materia prima y producto terminado para el ceviche de tilapia $(\mathrm{N}=25)$.

\begin{tabular}{ccccccc}
\hline \multirow{2}{*}{ Cantidad } & \multicolumn{2}{c}{ Recuento total } & \multicolumn{2}{c}{ Coliformes totales } & \multicolumn{2}{c}{ Coliformes fecales } \\
\cline { 2 - 6 } & $\begin{array}{c}\text { Materia prima } \\
\mathrm{n}(\%) \text { UFC/g }\end{array}$ & $\begin{array}{c}\text { Ceviche } \\
\text { UFC/g }\end{array}$ & $\begin{array}{c}\text { Materia prima } \\
\mathrm{n}(\%)(\mathrm{UFC} / \mathrm{g})\end{array}$ & $\begin{array}{c}\text { Ceviche } \\
(\mathrm{NMP} / \mathrm{g})\end{array}$ & $\begin{array}{c}\text { Materia prima } \\
\mathrm{n}(\%)(\mathrm{UFC} / \mathrm{g})\end{array}$ & $\begin{array}{c}\text { Ceviche } \\
(\mathrm{NMP} / \mathrm{g})\end{array}$ \\
\hline$<10$ & $2(8)$ & $11(42)$ & $2(8)$ & $9(36)$ & $19(76)$ & $12(48)$ \\
$10-1000$ & $0(0)$ & $4(15)$ & $2(8)$ & $13(52)$ & $4(16)$ & $11(44)$ \\
$>1000$ & $23(92)$ & $10(43)$ & $21(84)$ & $3(12)$ & $2(8)$ & $2(8)$
\end{tabular}

CUADRO 2

Comparación del recuento total psicrófilo aerobio, coliformes totales y coliformes fecales presentes en materia prima y producto terminado para el ceviche de camarón $(\mathrm{N}=27)$.

\begin{tabular}{ccccccc}
\hline & \multicolumn{2}{c}{ Recuento total } & \multicolumn{2}{c}{ Coliformes totales } & \multicolumn{2}{c}{ Coliformes fecales } \\
\cline { 2 - 7 } Cantidad & $\begin{array}{c}\text { Materia prima } \\
\mathrm{n}(\%) \text { UFC/g }\end{array}$ & $\begin{array}{c}\text { Ceviche } \\
\text { UFC/g }\end{array}$ & $\begin{array}{c}\text { Materia prima } \\
\mathrm{n}(\%)(\mathrm{UFC} / \mathrm{g})\end{array}$ & $\begin{array}{c}\text { Ceviche } \\
(\mathrm{NMP} / \mathrm{g})\end{array}$ & $\begin{array}{c}\text { Materia prima } \\
\mathrm{n}(\%)(\mathrm{UFC} / \mathrm{g})\end{array}$ & $\begin{array}{c}\text { Ceviche } \\
(\mathrm{NMP} / \mathrm{g})\end{array}$ \\
\hline$<10$ & $0(0)$ & $9(33)$ & $0(0)$ & $11(40,8)$ & $19(70,4)$ & $13(48,2)$ \\
$10-1000$ & $0(0)$ & $1(3)$ & $3(11)$ & $12(44,4)$ & $2(7,4)$ & $11(40,7)$ \\
$>1000$ & $27(100)$ & $17(64)$ & $24(89)$ & $4(14,8)$ & $6(22,2)$ & $3(11,1)$
\end{tabular}


en los respectivos ceviches. No se aisló ningún otro microorganismo potencialmente patógeno

Con respecto al camarón pelado, cabe destacar que el recuento total de aerobios psicrófilos presentes en la materia prima presentó una disminución significativa $(p<0,05)$ en el ceviche. No obstante, $74 \%$ de las muestras de camarón pelado presentaron un recuento total aerobio psicrófilo superior a $107 \mathrm{UFC/g}$.

Los coliformes totales también presentaron una importante disminución, variando de un $100 \%$ de positividad en materia prima a un $60 \%$ en el producto final. No obstante, la presencia de coliformes fecales fue detectada en $30 \%$ de la materia prima y $52 \%$ del producto final. En cuanto a la presencia de $E$. coli, en materia prima hubo un $12 \%$ de positividad, la cual disminuyó a $4 \%$ en el respectivo ceviche. De la misma manera, se obtuvo un aislamiento de Listeria monocytogenes a partir de la materia prima, la cual no fue aislada en el ceviche. Vibrio parahaemolyticus fue aislado a partir de 3 muestras de camarón y de 2 muestras del correspondiente ceviche. No se aisló Salmonella spp. ni $V$. cholerae de ninguna de las muestras evaluadas.
Los cuadros 3 y 4 muestran el comportamiento del ceviche de tilapia y ceviche de camarón, almacenado a $4^{\circ} \mathrm{C}$ durante una semana. Cabe destacar que el $\mathrm{pH}$ de ambos tipos de ceviche muestra una tendencia a la estabilidad o de aumento muy ligero. De la misma manera, la concentración de microorganismos de deterioro sufrió pocas variaciones. Es importante destacar el bajo número de Pseudomonas spp. presentes en el ceviche de tilapia así como la estabilidad del recuento de Enterococcus spp. a través de los días de almacenaje.

\section{DISCUSIÓN}

Los productos de pesquería son importantes no solo desde el punto de vista nutricional, sino también desde la perspectiva económica de los países productores y como un potencial riesgo a la Salud Pública (Yogoub 2009). En el presente trabajo, se analizó y correlacionó la carga microbiana inicial de pescado (tilapia) y camarón pelado con respecto a su producto final (ceviche).

La distribución porcentual de las muestras de pescado y camarón analizadas muestran que, la materia prima

CUADRO 3

Descripción de la variación del recuento total psicrófilo aerobio y de las poblaciones de Pseudomonas spp. y Enterococcus sp. presentes en ceviche de tilapia a través de su almacenamiento a $4^{\circ} \mathrm{C}$ por cinco días.

\begin{tabular}{ccccccc}
\hline Cantidad & \multicolumn{3}{c}{ Día 0 } & & Día 5 \\
\cline { 2 - 7 } & $\begin{array}{c}\text { Recuento total } \\
\text { aerobio psicrófilo } \\
\text { UFC/g n(\%) }\end{array}$ & $\begin{array}{c}\text { Recuento de } \\
\text { Pseudomonas spp. } \\
\text { UFC/g n(\%) }\end{array}$ & $\begin{array}{c}\text { Recuento de } \\
\text { Enterococcus sp. } \\
\text { UFC/g n(\%) }\end{array}$ & $\begin{array}{c}\text { Recuento total } \\
\text { aerobio psicrófilo } \\
\text { UFC/g n(\%) }\end{array}$ & $\begin{array}{c}\text { Recuento de } \\
\text { Pseudomonas } \\
\text { spp. UFC/g n(\%) }\end{array}$ & $\begin{array}{c}\text { Recuento de } \\
\text { Enterococcus } \\
\text { sp. UFC/g n(\%) }\end{array}$ \\
\hline$<100$ & $8(32)$ & $24(96)$ & $9(36)$ & $8(32)$ & $25(100)$ & $10(40)$ \\
$100-1000$ & $10(40)$ & $1(4)$ & $6(24)$ & $8(32)$ & 0 & $4(16)$ \\
$>1000$ & $7(28)$ & 0 & $10(40)$ & $9(36)$ & 0 & $11(44)$
\end{tabular}

\section{CUADRO 4}

Descripción de la variación del recuento total psicrófilo aerobio y de las poblaciones de Pseudomonas spp. y Enterococcus sp. presentes en ceviche de camarón a través de su almacenamiento a $4^{\circ} \mathrm{C}$ por cinco días.

\begin{tabular}{|c|c|c|c|c|c|c|}
\hline \multirow[b]{2}{*}{ Cantidad } & \multicolumn{3}{|c|}{ Día 0} & \multicolumn{3}{|c|}{ Día 5} \\
\hline & $\begin{array}{l}\text { Recuento total } \\
\text { aerobio psicrófilo } \\
\text { UFC/g } \mathrm{n}(\%)\end{array}$ & $\begin{array}{l}\text { Recuento de } \\
\text { Pseudomonas spp. } \\
\text { UFC/g } \mathrm{n}(\%)\end{array}$ & $\begin{array}{l}\text { Recuento de } \\
\text { Enterococcus sp. } \\
\text { UFC/g n(\%) }\end{array}$ & $\begin{array}{l}\text { Recuento total } \\
\text { aerobio psicrófilo } \\
\text { UFC/g } n(\%)\end{array}$ & $\begin{array}{c}\text { Recuento de } \\
\text { Pseudomonas } \\
\text { spp. UFC/g n(\%) }\end{array}$ & $\begin{array}{l}\text { Recuento de } \\
\text { Enterococcus } \\
\text { sp. UFC/g n(\%) }\end{array}$ \\
\hline$<100$ & $3(12)$ & $19(76)$ & $7(28)$ & $6(24)$ & $19(76)$ & $5(20)$ \\
\hline $100-1000$ & $6(24)$ & $4(16)$ & $2(8)$ & $3(12)$ & $3(12)$ & $3(12)$ \\
\hline$>1000$ & $16(64)$ & $2(8)$ & $16(64)$ & $16(64)$ & $3(12)$ & $17(68)$ \\
\hline
\end{tabular}


utilizada en la elaboración de ceviche posee un recuento elevado de microorganismos aerobios psicrófilos.

Al comparar los resultados de materia prima de tilapia con los parámetros establecidos por la International Comission for the Microbiological Examination of Foods (ICMSF) para pescado crudo, se encuentra que 15 muestras (60\%) sobrepasan el límite de $107 \mathrm{UFC/g}$, lo cual refleja que el producto en el momento de su análisis no presenta una frescura adecuada.

De manera contrastante, los productos finales presentan, con respecto a esta variable, valores significativamente más bajos, únicamente una de las muestras de ceviche de tilapia y cuatro de las muestras de ceviche de camarón presentaron recuentos totales aerobios psicrófilos superiores a $107 \mathrm{UFC/g}$ (Datos no mostrados). Resultados similares son reportados por Flores y colaboradores, quienes encuentran altos recuentos de psicrófilos en muestras de pescado, camarón y ceviche (Flores et al. 1996). Al respecto, ellos detallan que no existen normativas sanitarias para los ceviches, no obstante el recuento aerobio obtenido no es tan alto como el reportado a partir de los correspondientes alimentos crudos, justificándolo con base al marinado con ácidos de jugo de limón, naranja o vinagre al que se somete el ceviche (Flores et al. 1996). En el presente trabajo, el efecto de la disminución del pH es claro en la disminución de las cargas bacterianas obtenidas, pero tampoco se puede dejar de lado la posible presencia de agentes antimicrobianos químicos, tipo sorbatos y el efecto benéfico de la refrigeración en el control de poblaciones (Gelman et al. 2001).

Con respecto a los coliformes totales, es clara la disminución que se da al pasar de materia prima a producto terminado. Es evidente que ésta se debe principalmente a la variación del $\mathrm{pH}$ entre la materia prima $(\mathrm{pH}$ promedio $6,0-6,5)$ y los productos terminados ( $\mathrm{pH}$ promedio $4-5$ ). A pesar de la clara disminución en los coliformes totales, un alto porcentaje de los ceviches de tilapia y camarón presentan coliformes fecales en un nivel que evidencia mal manejo o contaminación post proceso.

La norma propuesta por la National Shellfish Sanitation Progam para coliformes fecales en camarón establece un máximo permitido de $2,3 / \mathrm{g}$, valor que se sobrepasa en $52 \%$ de los ceviches respectivos (NSSP 2009). De la misma manera, la ICMSF establece un límite de 5,0 x 102 de coliformes fecales / $g$ de pescado (ICMSF 1998), valor que se sobrepasa en $48 \%$ de los ceviches de tilapia evaluados. Lo anterior, aunado al hecho de que se aisló Escherichia coli a partir de $15 \%$ de ceviches de tilapia y $4 \%$ de los ceviches de camarón pone en evidencia el potencial riesgo que estos productos representan para la Salud Pública.
En el presente trabajo no se logró aislar Salmonella spp., datos similares a los reportados en el trabajo de Blanco y colaboradores, quienes únicamente lograron aislar una cepa a partir de una muestra de tilapia de origen costarricense. Lo anterior evidencia la buena calidad de las aguas utilizadas en la crianza de estos peces. A partir de camarón tampoco se aisló esta bacteria, lo cual coincide con los datos reportados por Flores y colaboradores en Yucatán (Flores et al. 1996) .

Se lograron aislar dos cepas de Vibrio parahaemolyticus a partir del ceviche de camarón (16\%), dato considerablemente superior a los resultados obtenidos por Flores et al. (Flores et al. 1996) quien describe un 2,1\% de positividad a partir de alimentos marinos y a los datos de Yagoub quien describe un $4,7 \%$ de aislamiento de esta bacteria a partir de pescado fresco vendido en mercados (Yagoub 2009). A pesar de que no se cuantifica esta bacteria, su aislamiento es importante dado que el producto podría ser conservado en condiciones que favorezcan la multiplicación de ésta.

No se aisló Listeria monocytogenes a partir de productos terminados, pero sí a partir de la materia prima. Con respecto al camarón, Embarek (1994) indica que es poco común el aislamiento de esta bacteria en agua de mar, a menos que la costa esté sujeta a contaminación proveniente de fuentes industriales, humanas o de animales, no obstante, su presencia en camarón pelado provenga probablemente de la manipulación o del ambiente de procesamiento. Con respecto a la tilapia, esta bacteria fue aislada a partir de 3 muestras, lo cual pone en evidencia una posible contaminación de las aguas de cultivo o un mal manejo del producto. Este resultado contrasta con lo reportado por Blanco y colaboradores, quienes no aislaron esta bacteria a partir de muestras de tilapia. Estudios similares realizados a partir de diversas especies de peces han proporcionado datos divergentes, presentándose amplias variaciones en los porcentajes de aislamiento. Karunasagar (2000) refiere que estas diferencias pueden deberse a la metodología utilizada, a diferencias en el tipo de muestra analizada (pescado fresco y fileteado) y a variaciones debidas a las distintas especies de peces o a la estación del año (Karunasagar 2000).

Con respecto a la variación del recuento total de psicrófilos aerobios y de las poblaciones de Pseudomonas spp. y Enterococcus spp. presentes en los ceviches de tilapia y camarón a través de su almacenamiento a $4^{\circ} \mathrm{C}$ por cinco días es importante destacar la poca variación que se da en estos, denotándose el efecto beneficioso de la refrigeración, de la acidez, así como la potencial presencia de agentes químicos en el control de poblaciones (Gelman et al. 2001). 
Con respecto a Pseudomonas, llama la atención el alto porcentaje de ceviches de tilapia y camarón con recuentos de $<100$ UFC/g de esta bacteria. Tal y como describe la literatura, el deterioro del pescado y productos marinos es asociada a tres factores: la autolisis, el crecimiento bacteriano y la oxidación química de los lípidos. Dentro de éstas, la actividad microbiológica es la variable que más afecta la calidad de éste. (Aberoumand 2010) y Pseudomonas spp. juega un papel primordial en el deterioro de estos tipos de alimentos (Koutsoumanis et al. 2000, Jeyasekaran et al. 2006).

Este bajo aislamiento de Pseudomonas spp. ha sido reportado por diversos autores, incluyendo a Yagoug (2009) y Aberoumand (2010), entre otros, quienes citan que el otoño representa el período del año en que se da el mayor aislamiento de esta bacteria y el verano el período en que se da el mayor porcentaje de aislamiento negativos, no solo para esta bacteria sino para la familia Enterobacteriaceae también y debido a la temperatura de las aguas. El presente trabajo fue realizado durante los meses de verano de Costa Rica, y al ser un país tropical, las temperaturas de sus aguas no descienden de manera significativa por lo que es de esperar resultados negativos.

Los resultados obtenidos a partir del análisis de materia prima y ceviches demuestran que, a pesar de la contaminación bacteriológica presente en la materia prima, y de que el ceviche es un producto que incluye mucha manipulación, existe una disminución importante en las cargas bacterianas en el producto final. No obstante, también se pone de manifiesto una importante contaminación fecal en productos listos para su consumo y a pesar de su bajo $\mathrm{pH}$; una situación indeseable y totalmente prevenible si se practican las adecuadas técnicas de manejo de alimentos, de higiene y se presta mayor atención a los puntos críticos de control de su formulación. No se debe de olvidar que las enfermedades de origen alimentario son consideradas por la FAO (Food and Agricultural Organization) y la OMS (Organización Mundial de la Salud) como el problema de salud con mayor distribución en el mundo y como una causa importante de la reducción de la productividad económica de las naciones (FAO/OMS 2000, Edema et al. 2005).

\section{REFERENCIAS}

Aberoumand, A. 2010. A review on Pseudomonas in marine fish. World Journal Fish Marine Science 2: 291-296.

Chacón, E. 2002. Biología general de la tilapia: curso básico de acuacultura, énfasis en trucha y tilapia. Instituto Tecnológico de Costa Rica, San Carlos, Alajuela, Costa Rica.
Edema, M.O., A.M. Omemu \& M.O. Bankole. 2005. Microbiological safety and quality of ready to eat foods in Nigeria. In Book of Abstract of the $29^{\text {th }}$ Annual Conference and General Meeting, Abeokuta. Nigerian Society Microbiology.

Embarek, B. 1994. Presence, detection and growth of Listeria monocytogenes in seafoods: a review. International Journal of Food Microbiology 23: 17-34.

FAO/OMS. 2000. Consulta Mixta de expertos sobre la evaluación de riesgos asociados a los peligros microbiológicos en alimentos. FAO/OMS, Roma, Italia.

FDA. 1995. Bacteriological and Analytical Manual. AOAC. Nueva York, Nueva York, EEUU.

Flores, J, G. Suárez, M. Heredia, M. Puc \& J. Monsreal. 1996. Calidad microbiológica de los alimentos marinos en la ciudad de Mérida, Yucatán. Veterinaria Mexicana 27: 319-324

Gelman, A, L. Glatman, V. Drabkin \& S. Harpaz. 2001. Effects of storage temperature and preservative treatment on shelf life of the pond raised freshwater fish, silver perch. Journal of Food Protection 64: 1584-1591

ICMSF. 1998. Microorganisms in foods: microbiological ecology of food contaminants. Blackie Academic and Professional, Londres, Inglaterra.

Jeyasekaran, G, P. Ganesan, R. Anandaraj, R. Jeya Shakila \& D. Sukumar. 2006. Quantitative and qualitative studies on the bacteriological quality of Indian White shrimp (Penaes indicus) stored in dry ice. Journal of Food Microbiology 23: 526-533

Karunasagar I. 2000. Listeria in tropical fish and fishery products. International Journal of Food Microbiology 62: 177-181.

Koutsoumanis, K \& G.J. Nychas. 2000. Application of systemic experimental procedure to develop a microbial model for rapid fish shelf life predictions. International Journal of Food Microbiology 60: 171-184.

Morales, G, L. Blanco, M.L. Arias \& C. Chaves. 2004. Evaluación de la calidad bacteriológica de tilapia fresca (Oreochromis niloticus) proveniente de la Zona Norte de Costa Rica. Archivo Latinoamericano en Nutrición 54: 433-437

National Shellfish Sanitation Program (NSSP). 2009. Guide for the Control of Molluscan Shellfish, EEUU.

Otárola, A. 2002. Producción acuícola en Costa Rica: curso básico de acuacultura, énfasis en trucha y tilapia. Instituto Tecnológico de Costa Rica, San Carlos, Alajuela, Costa Rica. 69-73.

Pascual, M, \& V. Calderón. 2000. Microbiología Alimentaria. Díaz de Santos, Madrid, España.

Pouch, F. 2001. Compendium of methods for the microbiological examination of foods. American Public Health Association 800 I St., NW Washington, DC. 343-353.

Ramírez, R. 2002. Aspectos de mercado nacional e internacional de la tilapia: Curso básico de acuacultura, énfasis en trucha y tilapia. Instituto Tecnológico de Costa Rica, San Carlos, Alajuela, Costa Rica: 74-80 
Shen, Y., Y. Liu, Y. Zhang, J. Cripe, W. Conway, J. Meng, G. Hall \& A. Bhagwat. 2006. Isolation and characterization of Listeria monocytogenes isolates from ready to eat foods in Florida. Applied and Environmental Microbiology 72: 5073-5074

Víquez, F, J. Aiello, C. Amerling. 2003. Características biométricas y químicas de la tilapia de agua dulce (Oreochromis nilotica) y uso del $\mathrm{pH}$ y de las características organolépticas para estimar su vida útil sensorial almacenada a $5^{\circ} \mathrm{C}$. Tesis. Universidad de Costa Rica.

Yagoub, S. 2009. Isolation of Enterobacteriaceae and Pseudomonas spp. from raw fish sold $\mathrm{n}$ fish market in Khartoum State. Journal of Bacteriological Research 1: 85-88 